Bol. Acad. peru. leng. 64. 2018 (27-46)

\title{
LA SIGLA, UN RECURSO EFICAZ EN LA PRENSA PERUANA
}

\section{ACRONYMS, AN EFFECTIVE RESOURCE IN THE PERUVIAN PRESS}

\author{
Eliana Gonzales Cruz \\ Universidad de Piura
}

\section{Resumen:}

Hoy en día el uso de las siglas resulta de gran interés e importancia debido a la predominancia que estas tienen en un escrito (periódicos, revistas, libros, informes, etc.); sin embargo, también podemos visualizar su empleo en los medios de comunicación audiovisual masivos como la radio y la televisión, en ambos aspectos se utiliza para identificar las denominaciones de las instituciones de todo tipo. Este uso se ve reflejado en la capacidad de síntesis unido al ahorro de espacio en cuanto a letras y palabras se refiere. En ese sentido, el presente estudio a través de un análisis descriptivo está orientado a explicar qué es lo que condiciona el uso de las siglas como recurso eficaz, pues al ser usadas o mencionadas en titulares pueden resultar ininteligibles para el lector u oyente por el desconocimiento que tienen en su significación o en cuanto a las reglas formales que se sigue para su construcción diferenciando su tipología. Por último, es importante resaltar que el uso de estas es más recurrente en el ámbito periodístico, ya que se dispone en estos medios espacios limitados en donde se está obligado a ajustarse a ellos. 


\section{Abstract:}

Nowadays the use of acronyms is of great interest and importance due to the predominance they have in written documents (newspapers, magazines, books, reports, etc.); however, we can also see their use in the mass audiovisual media such as radio and television, in both aspects they are used to identify the names of institutions of all kinds. This use is well reflected in the ability to synthesize and save space in terms of letters and words. In that sense, this study, through a descriptive analysis, is oriented to explain what conditions the use of acronyms as an effective resource, because when they are used or mentioned in headlines they can be unintelligible to the reader or listener due to the lack of awareness of their meaning or of the formal rules applied to their construction, distinguishing their typology. Finally, it is important to highlight that their use is more recurrent in the journalistic field, where there are limited spaces available and you are obliged to comply with them.

Palabras clave: Siglas, recurso eficaz, tipología, textos escritos.

Key words: Acronyms, effective resource, typology, written texts.

Fecha de recepción:

Fecha de aceptación:
$03 / 08 / 2018$

$31 / 10 / 2018$

\section{A modo de introducción}

Si bien la Academia señala que sigla es un «signo lingüístico formado con las letras iniciales de cada uno de los términos que integran una expresión compleja» (2010: 577) y, acrónimo, una sigla «cuya estructura se acomoda a los patrones silábicos característicos del español» (2010: 579); es importante precisar que coincido con el concepto de sigla, pero no con el de acrónimo, pues llamaré acrónimo a la combinación del inicio 
https://doi.org/10.46744/bapl.201802.002

y el final de dos palabras, por lo que serán siglas OTAN, CIA, FBI, ONU, ovni, sida, Unesco... y las incluyo en este trabajo; mientras que palabras como cantautor $(<$ cantante y autor), ofimática $(<$ oficina + informática), serán descartadas por acrónimos.

Cabe señalar, además, que dentro del grupo de las siglas distingo las propiamente dichas - lexicalizadas o no-, de los considerados sigloides (Casado Velarde, 1979: 72), llamados precisamente de esta manera porque no solo toman algunas letras iniciales, sino también algunos elementos conectores: UDEP (Universidad de Piura), Sunat (Superintendencia Nacional de Administración Tributaria), Fogem (Fondo de Garantía Empresarial).

Abordar el tema de las siglas en la prensa peruana responde a un interés personal motivado por titulares que pueden resultar ininteligibles a un lector promedio y, mucho más, a uno extranjero. Titulares como "Onagi usó dinero de rifas para comprar canastas navideñas a los gobernadores» (EC, 05-05-14), «¿AGP recibirá la carta del CEN?» (LR, 26-04-12), «PCM convoca a segundo concurso para presidentes de Osiptel y Ositrán» (LR, 29-05-12), «TC no invadió competencias del CNM y PJ, opinó legislador Carlos Eguren» (LR, 1310-13) $)^{1}$ me llevaron a plantearme preguntas como ies solo el factor espacio lo que condiciona el empleo de las siglas, de allí que se pueda considerar un recurso eficaz?, ¿se da con igual frecuencia en todos los medios escritos periodísticos?, ¿se ha intensificado últimamente o siempre han estado presentes?

Intentaré responder cada una de ellas en este estudio descriptivoanalítico y, a partir de ello, estableceré una tipología, haré un repaso de algunos aspectos relacionados con su escritura, describiré los principales rasgos morfológicos (género y número), analizaré el proceso de derivación, así como el grado de lexicalización de las siglas registradas

1 Según se recoge en OLE, las notae iuris o abreviaciones jurídicas existentes en el latín «proliferaron tanto que llegaron incluso a ser prohibidas en el siglo $\mathrm{V}$ porque su uso abusivo había llegado a dificultar gravemente la legibilidad de los textos (2010: 567). 
en algunos diarios peruanos, ${ }^{2} \mathrm{y}$, finalmente, determinaré en qué medida es un recurso eficaz.

\section{Aspectos formales}

Es sabido que en toda sigla podemos identificar dos partes: la base y la sigla propiamente dicha. La base, según Almela, es un complejo léxico constituido por un grupo de palabras que funciona como un bloque unitario (1999: 211). Así, en una sigla como Agalep, la base es Asociación de Ganaderos Lecheros del Perú. La normativa académica, en cambio, llama sigla «tanto al signo lingüístico formado con las letras iniciales de cada uno de los términos que integran una expresión compleja como a cada una de las letras iniciales» (OLE, 2010: 577).

Si bien actualmente se estila colocar la sigla en el titular de la noticia y la base en el cuerpo seguido de la sigla entre paréntesis, en décadas pasadas esto no sucedía así; pues se ha podido comprobar que algunas veces aparecía la sigla en el titular «Protesta por los maltratos elevó UNI al Poder Judicial» (LP, 25-05-60); y, luego, en el cuerpo se recogía solo la base, sin necesidad del referente siglar: «El Rector de la Universidad de Ingeniería... En la mañana se realizó en la sede de la Universidad de Ingeniería... Gino Ricci, Presidente de la Asociación de Centros de Estudiantes de la Universidad Nacional de Ingeniería...» (LP, 25-05-60).

El criterio del punto como marca distintiva ha ido variando con el tiempo. En la década del 60, era frecuente encontrarse con siglas que mantenían el punto, cosa que ya no ocurre actualmente «SE PREPARAN LOS BOXERS DEL C. J. N. B.», ${ }^{3}$ "Últimos acuerdos del C. N. de Deportes» (LP, 20-04-60); ${ }^{4}$ es más, en un mismo diario registramos siglas

2 Se han revisado los siguientes diarios peruanos: La Prensa, El Comercio, La República, Perú21, Expreso, Correo, Trome, Publimetro; de diferentes fechas. La selección se hizo de manera aleatoria.

3 Es importante precisar que se han mantenido las formas ortotipográficas tal y como aparecen en los diarios consultados.

4 Es probable que en este titular aún se vacile entre poner y no poner una sigla, pues consideramos que pudo aparecer CND para designar al Consejo Nacional de Deportes. 
https://doi.org/10.46744/bapl.201802.002

que llevan punto: «Los de A.C.F. Se Reunieron Secretamente» (LP, 2505-60) y las que no lo llevan: «La FEP Esta Noche a las 7 Hará Un Mitin de Protesta» (LP, 25-05-60). Nos hemos percatado también de que los puntos no aparecen en el titular (Respaldan en Ica Al Director de GUE San Luis» [LP, 23-05-60]), pero sí en el cuerpo de la noticia («Hasta la fecha le han manifestado su apoyo al Dr. Marroquín la Asociación de Profesores Secundarios de la G.U.E. «San Luis Gonzaga»» [LP, 23-05$60]$ ). Actualmente, los puntos han desaparecido por completo tal y como lo manda la norma. ${ }^{5}$

La vacilación de si va punto o no va probablemente se deba a que no se termina de distinguir de las llamadas abreviaturas que se caracterizan por llevar punto y espacio de separación: p. por página, Sr. por señor... y por marcar el plural, en algunos casos «duplicando la letra conservada ff. por folios, vv. por versos, ss. por siguientes, FF. AA. por Fuerzas Armadas» (2010: 573). ${ }^{6}$

El punto parece ser también el criterio de distinción entre el criptónimo de un personaje público de uno no público; así, no aparecen cuando se trata del criptónimo de personajes conocidos tales como AGP (Alan García Pérez), PPK (Pedro Pablo Kuczynski), JDC (Javier Diez Canseco), MVLL (Mario Vargas Llosa) y no MVLl como correspondería según la norma recogida en la Ortografía de la lengua española: "Cuando

5 Caso especial es el de PUTAS (Por una transformación auténtica de la sociedad), marcha organizada en Argentina. Al parecer, los medios quisieron dejar bien claro que se trataba de una sigla y acudieron para ello a los puntos: «La marcha de las P.U.T.A.S.: El deseo de ser respetadas y dejar de lado el machismo» (LR, 12-11-12).

6 EE. UU. es la abreviatura de Estados Unidos, aa. hh. lo es de asentamientos humanos y pp. ji. lo es de pueblos jóvenes. En todos los casos, el punto va pospuesto a cada bloque abreviado, nunca pospuesto a cada letra; de ahí que sea un error *E. E. U. U. (error que se han registrado en algunos diarios de 1960, de 1961, principalmente). La norma es precisa en señalar que se debe mantener el espacio en blanco entre los bloques; y que tampoco es correcto prescindir, como se hace la mayoría de las veces, del espacio entre los bloques (*EE.UU.) ni de los puntos, dejando el espacio (*EE UU), y menos aún escribir todo el conjunto sin puntos ni espacio (*EEUU) [OLE, 2010: 573]. Hemos podido comprobar que, en La República, medio de circulación nacional, en décadas pasadas (ochenta y noventa) sí los mantenía; pero actualmente, no. El Comercio, por su parte, en su libro de estilo, señala que es EE. UU. «(No EEUU ni USA)» (1998, 201). En este último medio, sí se conservan los puntos, pero no siempre el espacio. 
un dígrafo forma parte de una sigla, solo se escribe en mayúscula la primera de sus letras: COCh por Comité Olímpico de Chile» (2010: 584). ${ }^{7}$ Pero, cuando se trata de menores de edad agresores o agredidos, sus criptónimos suelen aparecer con puntos, así lo podemos ver en todos los diarios: «Según la Policía, "Gringasho» abrió fuego contra sus rivales, dejando heridos a tres de ellos, identificados como A.C.R. "Pinki» o «Chimbotano», K.P.V. «Chato Kevin» y CH.R.A. «Chocoto»» (C, 29 de abril de 2012), «Una niña de cuatro años, de iniciales M.P.R., fue violada por su vecino, de iniciales R.A.C., quien tiene 13 años» (P21, 23-01-12). En décadas pasadas también solía aparecer el criptónimo del autor de la noticia; ahora ya no. Es más, el libro de estilo de El Comercio señala que «Se prefiere el nombre completo, ya que el lector no está obligado a conocer las iniciales de todos los periodistas» $(1998: 116) .{ }^{8}$

Con respecto a la escritura, cabe precisar que no hay unanimidad en todos los medios, pues algunos mantienen las mayúsculas en todos los casos (UNICEF, ICPNA, IMARPE) y, en otros, aparece solo con la inicial siempre que resulte fácil su pronunciación: Unicef, Icpna, Imarpe, etc. ${ }^{9}$ El Comercio mantiene un claro criterio en su libro de estilo al señalar: «A partir de la quinta letra la sigla se escribe en altas y bajas. Cuando no se pueda pronunciar se escribirá en altas» (1998: 201). Esta vacilación no permite percibir claramente el proceso de lexicalización de una sigla; es el caso, por ejemplo, de VRAE, que aparece a veces en mayúsculas, y otras veces solo la mayúscula inicial como en Vrae, pero también vrae.

El grado de lexicalización es notorio en casos como pyme y mype que aparecen con marcas plurales (pymes y mypes); aunque algunas veces están escritas con letras mayúsculas y otras en minúsculas, predomina el uso en minúsculas. Otros casos peculiares serían mepecos (mediano

7 Hemos registrado también como parte del nombre del aeropuerto AIJCH (aeropuerto internacional Jorge Chávez).

8 En el diario Trome, los periodistas firman sus notas al final; algunas veces con nombre y apellidos completos, otras con la inicial del nombre y apellidos completos, y otras con un criptónimo, pero con puntos.

9 En la última ortografía académica, constituyen un grupo especial «aquellas cuya estructura permite leerlas como palabras (ovni, sida, OTAN, Unicef, etc.) y que se denominan, específicamente, acrónimos» (OLE, 2010: 565). 
https://doi.org/10.46744/bapl.201802.002

y pequeño contribuyente), pricos (principal contribuyente) y mipymes (micro, pequeña y mediana empresa), en donde la $s$ está ya integrada a la sigla.

Hablamos de lexicalización cuando una sigla se convierte en un lexema y es tratada como tal; es decir, se leen y funcionan como palabras: CEO («CEO del Scotiabank prevé que dólar seguirá a la baja» [LR, 4-5-12]), ONU, Unicef, radar, ovni, sida... El hablante, en la mayoría de los casos desconoce la composición sintagmática originaria. Así, los grados de lexicalización de una sigla están marcados por la pérdida de referencia de la base que no aparecen en el cuerpo de la noticia: MEF, Minedu, Minsa, Sunat, Confiep, Indecopi, Foncodes, Dincote, Sedapal, Sutep, Pronaa, Cofopri, Inabif; por el uso de las minúsculas: pyme, mype, vrae; por la presencia de la $s$ final como marca de plural: pymes, mypes; porque sirve de base de una nueva: RMV (Región Militar VRAE); y por el proceso de derivación: apristas, fonavistas, sutepistas, claeístas, udepinos.

Se ha podido comprobar que, principalmente, los sufijos que se adjuntan a una base síglica son -ista: aprista (de Apra), pepecista (de PPC), fonavista (de Fonavi), claeísta (de CLAE), emerretista (de MRTA), sutepista (de Sutep), regetepista (de CGTP); e -ismo: aprismo (de Apra), pepecismo (de $P P C$ ). El primero suele indicar los miembros de la institución designada por la sigla o el sigloide y aparece principalmente con partidos políticos (Apra, PPC), instituciones sindicales (Sutep, CGTP). En menor cantidad y, sobre todo, últimamente se está añadiendo el sufijo fobia a la sigla LGTB (LGTBfobia). Al respecto, es importante señalar que esta sigla es de las pocas que ha ido aumentando; de LGTB pasó a LGTBI, luego a LGTBIQ; y últimamente también existen LGTBIQP, LGTBIQPA, LGTTBIQPA; ${ }^{10}$ e incluso LGTB +.

Hay casos en los que las siglas aparecen prefijadas, pero en menor número: PRE-ESNAV (Centro de Preparación de la Escuela Naval), Pre

10 La sigla LGTTBIQPA correspondería a lesbianas, gais, transexuales, transgéneros, bisexuales, intersexuales, queer o cuir (que no pertenece a ninguno), pansexuales y asexuales. 
Usil (Centro de Estudios Pre Universitarios de la Universidad San Ignacio de Loyola), Sub-CAFAE (Sub comité de administración de fondo de asistencia y estímulo). En estos casos, los respectivos prefijos no han sido añadidos a la sigla después de su creación, sino que aparecen formando parte de ella. No es el caso de anti-OTAN, proUNESCO ni de exPIP (ex miembro de la Policía de Investigaciones del Perú).

Por otro lado, cabe señalar que la expresividad aparece cuando se hace coincidir algunas siglas con palabras bastante conocidas; así, no hay mejor nombre para impulsar la actividad empresarial en Gamarra que Unete; o incluso llamar ELSA a una embotelladora nacional con alcance internacional. Incluimos también siglas tales como CADA (Congreso Anual de Alcaldes), CAEN (Centro de Altos Estudios Nacionales), CRAC (Cajas Rurales de Ahorro y Crédito), TASA (Tecnológica de Alimentos Sociedad Anónima), ANDA (Asociación Nacional de Anunciantes), Ahora (Asociación de hoteles, restaurantes y afines), CUNA (Congreso Unitario Caional Agrario) y PUTAS (Por una transformación auténtica de la sociedad). En este último caso, los medios quisieron dejar bien claro que se trataba de una sigla y acudieron para ello a los puntos: «La marcha de las P.U.T.A.S.: El deseo de ser respetadas y dejar de lado el machismo» (LR, 12-11-12).

Pero no solo la expresividad, sino incluso el ingenio regional es lo que se mostró en algunos de los partidos y asociaciones que surgieron durante los dos últimos periodos electorales: FIRME (Frente de Integración Regional Moquegua Emprendedora), MAS (Movimiento Andino Socialista/Movimiento de Afirmación Social), PAPÁ (Patria Arriba Perú Adelante), PICO (Proyecto Integracionista de Comunidades Organizadas), MIRA (Movimiento de Integración Regional Amazónico), CAMU (Ciencia y Acción Movilizadora de Ucayali), este último en clara alusión al camu camu, la planta nativa de la Amazonía peruana altamente apreciada por la importante concentración de vitamina C.

En nuestra recolección hemos encontrado algunas que comparten la misma sigla: CLAE fue la sigla del Centro Latinoamericano de Asesoramiento Empresarial; ahora es la sigla del Consorcio Latinoamericano 
https://doi.org/10.46744/bapl.201802.002

de Anticoncepción de Emergencia. Otros casos: AAA sigla del programa televisivo Amor, amor, amor; de la Asociación de Alcohólicos Anónimos; y de la Asociación de Artistas Aficionados; CNI fue en Consejo Nacional de Inteligencia; pero es también la sigla del equipo de fútbol Colegio Nacional de Iquitos.

Hay casos en los que se mezclan tanto la abreviación como la siglación; así en CCFFAA tenemos que las dos primeras letras corresponden a Comando Conjunto y las siguientes a Fuerzas Armas; lo mismo que en CNDDHH; las dos primeras son de Coordinadora Nacional, y las siguientes de Derechos Humanos. ${ }^{11}$

Por otro lado, cabe señalar que, si bien la normativa es clara en señalar que las siglas no llevan tildes, se ha notado una clara vacilación entre ADEX y Ádex, Comex y Cómex, Corpac y Córpac, Ositran y Ositrán. Unas veces aparecen con tilde y otras sin. Esto puede ser un claro paso hacia la lexicalización. En el caso de Unifé (Universidad Femenina del Sagrado Corazón), esta vacilación desaparece porque siempre se escribe con tilde, por lo que es un claro ejemplo de lexicalización.

Hemos registrado casos en los que se conculca la regla que obliga a escribir $m$, en lugar de $n$, ante $p$ en las siglas ONP (Oficina de Normalización Previsional), ONPE (Oficina Nacional de Procesos Electorales) e INPE (Instituto Nacional Penitenciario). Aunque en la mayoría de los casos se mantienen en mayúsculas, como lo señala la norma (Ortografía, 2010: 584), hay diarios que solo conservan la mayúscula inicial: «Inpe investiga fuga de reos» (E, 30-04-12).

Con respecto al género, sabemos que está determinado por el género de la palabra que hace de núcleo de la base; así, tenemos: la Sunat (por Superintendencia), el JNE (por Jurado), la UNMSM (por Universidad), etc. La a tónica no condiciona el cambio de artículo; así, es la $\mathrm{ABE}$ y no el $\mathrm{ABE}$ porque se trata de la Asociación de Buenos

11 También se ha registrado CIDH por Consejo Interamericano de Derechos Humanos, pero también para Corte Interamericana de Derechos Humanos (LR, 20-4-12). Una misma sigla para dos instancias. 
Empleadores. Caso contrario es el que ocurre con APRA, pues a pesar de ser Alianza Popular Revolucionaria Americana se suele usar el APRA al parecer porque en el colectivo de la gente está presente la forma partido más que alianza. ${ }^{12}$ Ahora bien, con respecto a la sigla U de Club Universitario de Deportes, no se dice el U por el Club, sino «la U» por el género femenino de letra. Es la única sigla que mantiene las comillas dobles o las simples, tanto en los titulares como en el cuerpo de la noticia: «Botafogo Da Mañana La Revancha a La «U»» (LP, 5-07-60); «Alianza y la 'U' dejan su crisis para jugar» (EC, 15-04-12).

En cuanto al número, la norma señala que el plural se marca mediante los determinantes plurales: algunas ONG, tres DNI, varias CMAC. Si bien esta es la forma que predomina, no es extraño encontrarse con formas tales como AFPs, AFP's, CDs, CD's.

Es bastante sabido que el empleo de siglas es un fenómeno muy extendido en casi todos los ámbitos. Su uso no solo responde a la idea de reducir o abreviar a una sola «pieza léxica» (lexía) expresiones complejas que continuamente son empleadas. Ya lo dijo Manuel Casado hace más de una década: «Se cuentan por decenas el número de siglas en cualquier periódico o revista de actualidad» (1999: 5081). En efecto, he podido registrar varios cientos, lo que me ha permitido hacer un esbozo tipológico teniendo como referencia distintos criterios tales como los siguientes:

\section{a. La cantidad de letras}

- unilíteras (U),

- bilíteras (AP), (PJ),

- trilíteras (AFP),

- tetralíteras (MRTA),

- pentalíteras (Sunat),

12 Si bien en la prensa hay un cuidado por mantener el masculino en Reniec (el Reniec), no ocurre lo mismo en el plano oral que predomina la Reniec. Los hablantes establecen una «falsa concordancia» con oficina, e incluso, con institución y no con registro. Cfr. la recomendación de Castellano Actual que aparece en http://udep.edu.pe/castellanoactual/ es-la-reniec-o-el-reniec/ 
https://doi.org/10.46744/bapl.201802.002

- hexalíteras (Agalep),

- heptalíteras (Aidesep),

- octolíteras (Alafarpe),

- nonalíteras (Apetrices),

- decalíteras (Fenexdrelp),

- undecalíteras (Conafovicer).

\section{b. La composición interna}

- alfabéticas (ONPE, INPE),

- alfamatemáticas (EPL2R, CxC).

\section{c. La pronunciación}

- silábicas porque se pronuncian de manera continuada, seguida (ADEX, Capeco, RUC);

- consonánticas porque se deletrean una por una (AFP, CGTP, BCR) y

- mixtas porque se mezclan las dos formas (ACNUR, CPAL, PNUD).

\section{d. La naturaleza compositiva}

- propias porque solo toma la letra inicial (CPC, DREM, FNG) e

- impropias o sigloides porque no toman solo la primera letra (Fonare, Inabif), sino incluso los conectores (UDEP, pyme) o la letra inicial de los conectores (Sedapal).

\section{e. Los ámbitos}

- política (PPC, AP, Frenatraca);

- gobierno:

ministerios (Mindef, Minag, Minsa),

$>$ fuerzas armadas (PNP, FAP, EP, Dinandro, Dirincri, Diroes);

programas sociales (Pronaa, Pronama, Pronasar);

$>$ organismos (INPE, ONPE, OCMA, Sunat, Indecopi);

- centros de estudios:

universidades (PUCP, UNMSM, USIL, UPCH, UARM);

$>$ dependencias universitarias (CUM) 
https://doi.org/10.46744/bapl.201802.002

procesos de admisión (TAA, PAE)

asignaturas (LE, HP, MB)

institutos (ETS, ISA);

- empresas y servicios (Edelnor, Sedapal, CMAC, BCP);

- agrupaciones:

$>$ asociaciones (APA, Apafa, Ahora, Adex);

corporaciones (Cofide, CCCA);

sindicatos (Sutep, CGTP, Sinamssop);

colegios profesionales (CAL, CMP);

- marcas comerciales (BVD, KR, GN, ENCI);

- federaciones deportivas (FPF);

- programas televisivos (BDP, AFHS);

- canales y emisoras (RPP, CPN, ATV);

- fenómenos meteorológicos (FEN);

- delimitaciones territoriales:

valles, regiones (VRAE)

distritos: SJL (San Juan de Lurigancho), SJM (San Juan de Miraflores), VES (Villa El Salvador)

\section{A modo de conclusión}

A modo de conclusión, puedo decir que el uso exagerado de las siglas en la prensa peruana está condicionado por la economía del espacio y por la carrera contra el tiempo que, al parecer, es el rasgo de nuestra modernidad. Si no hay espacio para colocar el nombre completo, tampoco hay tiempo para leerlo, de ahí que uno se pueda encontrar con hasta tres siglas en los titulares y con hasta ocho o nueve en el cuerpo de la noticia. Esto, sin duda, confirma que estamos ante un recurso eficaz.

A esto añadimos que hay una mayor tendencia a consagrarlas como palabras de uso cotidiano, tendencia que se ve reflejada en el uso oral en diferentes registros comunicativos, como hemos podido notar en el esbozo tipológico, pues si bien su empleo empieza hacia la década del treinta, este no se ha detenido; al contrario, podemos afirmar que se ha intensificado en su gran mayoría como nombres propios; sin embargo, encontramos con un uso adjetival: general 
https://doi.org/10.46744/bapl.201802.002

PNP, comandante FAP, región PNP. Asimismo, observamos que hay una mayor tendencia a la lexicalización, especialmente de siglas del ámbito gubernamental: Minedu, Minsa, Sunat, PJ, Foncodes; otras, en cambio, como Sinamos, Pronaa, Banmat, ENCI, ANR, AFHS... han ido desapareciendo porque su significación estuvo circunscrita a un momento histórico-cultural determinado. Esto último confirma lo que han destacado los especialistas: que las siglas nacen y mueren al mismo tiempo que surgen y desaparecen las instituciones o referencias que las designan.

Finalmente, me gustaría terminar insistiendo que existe un gran desconocimiento de las reglas formales de utilización de siglas por parte de los periodistas; para algunos quizás no es clara la distinción entre sigla y abreviatura, de allí que se suelan cometer muchos errores que hemos ido señalando. Se percibe un constante descuido lo que desvirtualiza las marcas de lexicalización en la mayoría de ellas; sin embargo, es preciso reconocer que algunos medios son más cuidadosos que otros, no solo en el uso ortográfico, también en el gramatical.

\section{Siglas utilizadas}

AAA Amor, amor, amor; Asociación de Alcohólicos Anónimos; Asociación de Artistas Aficionados;

ABE Asociación de Buenos Empleadores

ACF Asociación Central de Fútbol

ACNUR Alto Comisionado de las Naciones Unidas para los Refugiados

ADEX Asociación de Exportadores

AFHS Al fondo hay sitio

AFP Administradora de fondo de pensiones

Agalep Asociación de Ganaderos Lecheros del Perú

AGP Alan García Pérez

Ahora Asociación de hoteles, restaurantes y afines

Aidesep Asociación Interétnica de Desarrollo de la Selva Peruana

Alafarpe Asociación Nacional de Laboratorios Farmacéuticos del Perú 
https://doi.org/10.46744/bapl.201802.002

ANR Asamblea Nacional de Rectores

AP

Acción Popular

APA

Asociación Peruana de Avicultura

Apafa

Asociación de Padres de Familia

Apetrices

Asociación Peruana de Productores de Trigo y Cebada

APRA

Alianza Popular Revolucionaria Americana

ATV

Andina de Televisión

Banmat

Banco de Materiales

BCR

Banco Central de Reserva

BDP

Buenos días, Perú

BRAE

Boletín de la Real Academia Española

BVD

Bradley, Voorhees \& Day

CADA

Congreso Anual de Alcaldes

CAEN

Centro de Altos Estudios Nacionales

CAFAE

Comité de Administración de Fondo de Asistencia y Estímulo

CAL

Colegio de Abogados de Lima

CAMU Ciencia y Acción Movilizadora de Ucayali

Capeco Cámara Peruana de la Construcción

CCCA

Corporación del Cuero, Calzado y Afines

CCFFAA Comando Conjunto de las Fuerzas Armas

$\mathrm{CD}$

Compac Disc (disco compacto)

CEN

Comité Ejecutivo Nacional

CEO

Chief Operating Officer

CGTP

Confederación General de Trabajadores del Perú

CIA

Central Intelligence Agency (Agencia Central de Inteligencia)

CJNB Círculo Juventud Nacional de Box

CLAE Centro Latinoamericano de Asesoramiento Empresarial;

Consorcio Latinoamericano de Anticoncepción de Emergencia

CMAC Caja Municipal de Ahorro y Crédito

CMP Colegio Médico del Perú

$\mathrm{CN} \quad$ Comité Nacional

CNDDHH Coordinadora Nacional de Derechos Humanos

$\mathrm{CNI}$

Consejo Nacional de Inteligencia; Colegio Nacional de Iquitos 
https://doi.org/10.46744/bapl.201802.002

Cofide Corporación Financiera de Desarrollo

Cofopri Comisión de la Formalización de la Propiedad Informal

Comex Comercio Exterior

Conafovicer Comité Nacional de Administración del Fondo para la Construcción de Vivienda y Centros Recreacionales

Confiep Confederación Nacional de Instituciones Empresariales Privadas

Corpac Corporación Peruana de Aeropuertos y Aviación Comercial

CPAL Centro Peruano de Audición y Lenguaje

CPC Comisión de Protección al Consumidor

CPN Contaduría Pública de la Nación; Cadena Peruana de Noticias

CRAC Cajas Rurales de Ahorro y Crédito

CUM Centro Universitario de Medicina Preventiva

$\mathrm{CxC} \quad$ Ciudadanos por el Cambio

Dinandro Dirección Nacional Antidrogas

Dincote Dirección contra el Terrorismo

Dirincri Dirección de Investigación Criminal

Diroes Dirección de Operaciones Especiales

DNI Documento nacional de identidad

DREM Dirección Regional de Energía y Minas

Edelnor Empresa de Distribución Eléctrica de Lima Norte

Elsa Embotelladora Latinoamericana Sociedad Anónima

ELUA Estudios de Lingüística Universidad de Alicante

ENCI Empresa Nacional de Comercialización de Insumos

EP Ejército Peruano

EPL2R Explorar, preguntar, leer, recitar y repasar

ESNAV Escuela Naval

ETS Escuela Tecnológica Superior; enfermedad de transmisión sexual

FAP Fuerza Aérea Peruana

FBI Federal Bureau of Investigation (Oficina Federal de Investigaciones)

FEN Fenómeno de El Niño

Fenexdrelp Federación Nacional de Expendedores de Diarios, Revistas y Loterías del Perú 
https://doi.org/10.46744/bapl.201802.002

$\begin{array}{ll}\text { FEP } & \text { Federación de Estudiantes del Perú } \\ \text { FIRME } & \text { Frente de Integración Regional Moquegua Emprendedora } \\ \text { FNG } & \text { Frente Nacional Ganadero } \\ \text { Fogem } & \text { Fondo de Garantía Empresarial } \\ \text { Fonare } & \text { Foro Nacional de Reciclaje } \\ \text { Fonavi } & \text { Fondo Nacional de Vivienda } \\ \text { Foncodes } & \text { Fondo Nacional de Cooperación para el Desarrollo } \\ \text { FPF } & \text { Federación Peruana de Fútbol } \\ \text { Frenatraca } & \text { Frente Nacional de Trabajadores y Campesinos } \\ \text { GN } & \text { Galletas del Norte } \\ \text { GUE } & \text { Gran Unidad Escolar } \\ \text { HP } & \text { Historia del Perú, Hewlett-Packard } \\ \text { Icpna } & \text { Instituto Cultural Peruano Norteamericano } \\ \text { Imarpe } & \text { Instituto del Mar Peruano } \\ \text { Inabif } & \text { Instituto Nacional de Bienestar Familiar } \\ \text { Indecopi } & \text { Instituto Nacional de Defensa de la Competencia y de la } \\ & \text { Protección de la Propiedad Intelectual } \\ \text { INPE } & \text { Instituto Nacional Penitenciario } \\ \text { ISA } & \text { Instituto Santa Ángela } \\ \text { JDC } & \text { Javier Diez Canseco } \\ \text { JNE } & \text { Jurado Nacional de Elecciones } \\ \text { KR } & \text { Kola Real } \\ \text { LE } & \text { Lengua Española } \\ \text { LEA } & \text { Lingüística española actual } \\ \text { MAS } & \text { Movimiento Andino Socialista, Movimiento de Afirmación } \\ & \text { Social } \\ \text { MB } & \text { Matemática Básica } \\ \text { mepecos } & \text { mediano y pequeño contribuyente } \\ \text { Minag } & \text { Ministerio de Agricultura } \\ \text { Mindef } & \text { Ministerio de Defensa } \\ \text { Minsa } & \text { Ministerio de Salud } \\ \text { Mipymes } & \text { Micro, pequeña y mediana empresa } \\ \text { MIRA } & \text { Movimiento de Integración Regional Amazónico } \\ \text { MRTA } & \text { Movimiento Revolucionario Túpac Amaru } \\ \text { MVLl } & \text { Mario Vargas Llosa } \\ \text { mype } & \text { Micro y pequeña empresa } \\ & \end{array}$


https://doi.org/10.46744/bapl.201802.002

OCMA Oficina de Control de la Magistratura

OLE Ortografía de la lengua española

ONG Organismo no gubernamental

ONP Oficina de Normalización Previsional

ONPE Oficina Nacional de ProcesOrganización de las Naciones Unidas

Osiptel Organismo Supervisor de Inversión Privada de Telecomunicaciones

Ositran Organismo Supervisor de la Inversión en Infraestructura de Transporte de Uso Público

OTAN Organización del Tratado Atlántico Norte

Ovni objeto volador no identificado

PAE Prueba de Aptitud Escolar

PAPÁ Patria Arriba Perú Adelante

PCM Presidencia de Consejo de Ministros

PICO Proyecto Integracionista de Comunidades Organizadas

PIP Policía de Investigaciones del Perú

PJ Poder Judicial

PNP Policía Nacional del Perú

PNUD Programa de las Naciones Unidas para el Desarrollo

PPC Partido Popular Cristiano

PPK Pedro Pablo Kuczynski

Pricos Principal contribuyente

Pronaa Programa Nacional de Asistencia Alimentaria

Pronama Programa Nacional de Movilización por la Alfabetización

Pronasar Programa Nacional de Agua y Saneamiento Rural

PUCP Pontificia Universidad Católica del Perú

Putas Por una transformación auténtica de la sociedad

pyme pequeña y mediana empresa

radar Radio Detecting and Ranging

RAE Real Academia Española

RMV Región Militar VRAE

RPP Radio Programas del Perú

RUC Registro único de contribuyente

Sedapal Servicio de Agua Potable y Alcantarillado de Lima

sida Síndrome de inmunodeficiencia adquirida 
https://doi.org/10.46744/bapl.201802.002

$\begin{array}{ll}\text { Sinamos } & \text { Sistema Nacional de Movilización Social } \\ \text { Sinamssop } & \text { Sindicato Nacional Médico del Seguro Social del Perú } \\ \text { SJL } & \text { San Juan de Lurigancho } \\ \text { SJM } & \text { San Juan de Miraflores } \\ \text { Sunat } & \text { Superintendencia Nacional de Administración Tributaria } \\ \text { Sutep } & \text { Sindicato Unitario de Trabajadores de la Educación del } \\ & \text { Perú } \\ \text { TAA } & \text { Test de Aptitud Académica } \\ \text { TASA } & \text { Tecnológica de Alimentos Sociedad Anónima } \\ \text { U } & \text { Club Universitario de Deportes, } \\ \text { UARM } & \text { Universidad Antonio Ruiz de Montoya } \\ \text { UDEP } & \text { Universidad de Piura } \\ \text { Unesco } & \text { United Nations Educational, Scientific and Cultural } \\ & \text { Organization (Organización de las Naciones Unidas para } \\ \text { Unete } & \text { la Educación, la Ciencia y la Cultura) } \\ \text { Uni } & \text { Unidad de Empresarios Textiles de Gamarra } \\ \text { Unicef } & \text { United Nations Children's Fund (Fondo de las Naciones } \\ & \text { Unidas para la Infancia) } \\ \text { Unifé } & \text { Universidad Femenina del Sagrado Corazón } \\ \text { UNMSM } & \text { Universidad Nacional Mayor de San Marcos } \\ \text { UPCH } & \text { Universidad Peruana Cayetano Heredia } \\ \text { USA } & \text { United States of America } \\ \text { USIL } & \text { Universidad San Ignacio de Loyola } \\ \text { VES } & \text { Villa El Salvador } \\ \text { VRAE } & \text { Valle de los ríos Apurímac y Ene }\end{array}$


https://doi.org/10.46744/bapl.201802.002

\section{BIBLIOGRAFÍA}

ALBA DE DIEGO, V. (1973). «Marcas, abreviaciones y siglas en el lenguaje publicitario», Prohemia 4: 348-378.

ALMELA, R. (1999). Procedimientos de formación de palabras, Barcelona: Ariel.

ALONSO, D. (1962). «La invasión de las siglas», Del siglo de oro a este siglo de las siglas, Madrid: Gredos.

ALVAR EZQUERRA, M. y MIRÓ DOMÍNGUEZ, A. (1983). Diccionario de siglas y abreviaturas, Madrid: Alhambra.

ALVAR EZQUERRA, M. (1996). La formación de palabras en español, Madrid: Arco Libros.

CASADO VELARDE, M. (1979). «Creación léxica mediante siglas», Revista Española de Lingüística 9, vol. 1, 67-88.

. (1999). «Otros procesos morfológicos: acortamientos, formación de siglas y acrónimos», en Bosque, I. y V. Demonte, Gramática Descriptiva del español 3, Madrid: Espasa Calpe, 5075-5096.

GONZÁLEZ OLLÉ, F. y CASADO VELARDE, M. (1992). «Formación de palabras», Lexikon der Romanistischen Linguistik VI: 1, Tubinga: Max Niemeyer, 91-109.

LANG, M. (1992). Formación de palabras en español. Morfología derivativa productiva, Madrid: Cátedra.

MARTÍN BOSQUE, A. (2003). «Español / italiano. El tratamiento de las siglas en la prensa escrita», Mots Palabras Words 4, 75-92. 
https://doi.org/10.46744/bapl.201802.002

MARTÍNEZ DE SOUSA, J. (1984). Diccionario internacional de siglas y acrónimos, Madrid: Pirámide, 1984.

Real Academia de la Lengua Española y Asociación de Academias de la Lengua Española. (2010). Ortografía de la lengua española, Madrid: Espasa Libros.

RODRÍGUEZ GONZÁLEZ, F. (1989a). «La derivación de las siglas», BRAE LXIX, CCXLVII, 211-255.

. (1989b). «Las siglas como procedimiento lexicogenésiCO», ELUA 9, 9-24.

VARELA ORTEGA, S. (2005). «Otros procedimientos de formación de palabras: Acortamientos, siglas y acrónimos», Madrid: Gredos, 89-101.

WITTLIN, C. (1981). «Un nuevo tipo de siglas: acrónimos lexemas contextuales», LEA III: 1, págs. 159-174. 